\title{
Wound-healing activity of immobilized postbiotics from Bacillus subtilis exometabolites
}

\author{
Dina Shokatayeva*, Irina Savitskaya, and Aida Kistaubayeva \\ Al-Farabi Kazakh National University, 050040, Almaty, Kazakhstan
}

\begin{abstract}
The biological activity of postbiotic from Bacillus subtilis exometabolites was determined due to protein content $-0.541 \pm 13.4$ $\mathrm{mg} / \mathrm{ml}$, the level of proteases $-7.8 \pm 0.3 \mathrm{U} / \mathrm{ml}$ and the presence of antimicrobial substances. A biocomposite material was developed by coaggregation of bacterial cellulose, chitosan and Bacillus exometabolites. Modified BC gel film possesses high antagonistic activity against causative agents of wound infections: Staphylococcus aureus, Escherichia coli, Pseudomonas aeruginosa, Staphylococcus epidermidis. The use of the obtained material in treatment of wounds on laboratory animals reduces healing time by an average of $20 \%$. The developed bioactive wound dressings is intended for local application in order to optimize the wound healing process.
\end{abstract}

Keywords: Bacillus subtilis, postbiotics, antagonistic activity.

\section{Introduction}

Studies on the production and application of dozens of variants of wound dressings based on natural polymers are described in numerous scientific and patent reports in recent years [1-2]. The advantages of natural polymeric materials are their biocompatibility, non-toxicity, and a controlled biodegradation process.

Bacterial cellulose (BC) membranes show a rapid rate of epithelialization and tissue regeneration during wound healing. The $\mathrm{BC}$ gel film is a matrix - a carrier of almost any drug and, thus, can be used as a universal wound dressing for various types of injuries, including diabetic wounds on legs, chronic, mechanical wounds and burns [3].

The choice of medicinal components in the carrier matrix, providing dosed and prolonged release of the latter into the wound, while maintaining its sorption capacity, is an urgent issue.

In accordance with the planned tasks, a study was carried out to create a biocomposite consisting of three components: a cellulose matrix, chitosan and Bacillus subtilis exometabolites.

Chitosan $(\mathrm{Ch})$ belongs to connective tissue glycopolysaccharides, which are part of the extracellular matrix. It is close to the functional qualities of dermis [4]. This aminopolysaccharide enhances the processes of damaged tissue regeneration, is nontoxic, prevents the blood clots formation, therefore it is often used to create film materials in tissue engineering [5]. Protective wound dressings made of chitosan are air and vapor permeable, prevent the invasion of wound by non-desirable microorganisms, create an optimal microclimate in the wound, and promote cell proliferation [4].

*Corresponding author: dina_ibrayeva_91@mail.ru 
Bacteria of the Bacillus genus produce postbiotics - metabolic products that have biological activity in relation to the host organism. The production of a wide range of antibiotics and proteolytic enzymes by bacteria of the Bacillus genus, stimulating tissue regeneration processes, is the reason for studying the possibility of using the metabolites of these bacteria for functionalization of wound dressings based on BC gel film [6].

The inclusion of biologically active components in the $\mathrm{BC}$ polymer will make it possible to influence the two most important etiological links of the wound process necrotic tissues and microbial contamination.

Aim: development of a wound-healing biocomposite material with antimicrobial properties by including chitosan and Bacillus exometabolites in the bacterial cellulose.

\section{Materials and methods}

\subsection{Microbial strains}

Komagatei bacter xylinus C-3 and Bacillus subtilisP-2 strains were isolated at the Biotechnology Department, Al-Farabi Kazakh National University; the cultures were deposited in Republic Collection of Microorganisms (Astana, Kazakhstan); Gen Bank accession numbers are KU598766 and KY780502, respectively.

Esherichia coli ATCC 8799, Pseudomonas aeruginosa ATCC 9027, Staphylococcus aureus ATCC 6538, Staphylococcus epidermidis ATCC 14990 test cultures were obtained from American type culture collection.

\subsection{Production and preparation of BC films}

The production of BC films by Komagatei bacter xylinusC-3was carried out using modified Hestrin-Shramm medium with the addition of $0.5 \%$ ethanol and $0.1 \%$ beer wort. Cultivation was carried out at $29-30^{\circ} \mathrm{C}$ for 6-7 days. The developed gel-like cellulose pellicle was first purified by washing with deionized water for 5-7 minutes. Then it was treated with $1 \%(\mathrm{w} / \mathrm{v})$ $\mathrm{NaOH}$ at $35^{\circ} \mathrm{C}$ for $24 \mathrm{~h}$ to remove bacterial cells and the obtained acellular matrix was rinsed with deionized water until the $\mathrm{pH}$ of the rinsing solution was 6.8-7.2. BC films were sterilized by autoclaving.

\subsection{Immobilization of metabolites of the B. subtilis culture fluid supernatant on $\mathrm{BC} / \mathrm{Ch}$ matrix}

Separation of native microbial culture was carried out in an MPW-350 centrifuge for 30 minutes at a 10,000 rpm speed. Residual cells were present in the supernatant; therefore, it was passed through a membrane filter to remove them. For control, the obtained cultural liquid was plated on potato agar. Pieces of $\mathrm{BC}$ film were placed into the cell-free supernatant and incubated at room temperature $\left(25^{\circ} \mathrm{C}\right)$ under dynamic conditions $(80 \mathrm{rpm})$ for 5 minutes. Then $0.5 \mathrm{ml}$ of a $2.5 \%$ glutaraldehyde solution was added and kept at $30{ }^{\circ} \mathrm{C}$ for 2 hours at $100 \mathrm{rpm}$. To complete the immobilization, the $\mathrm{BC}$ films were washed several times with phosphate buffer ( $\mathrm{pH} 5.2,200 \mathrm{mM})$ and dried.

\subsection{Determination of proteases in the B. subtilis P-2 cell-free culture fluid supernatant}

A $0.5 \%$ solution of azocasein was prepared in $0.05 \mathrm{M}$ Tris- $\mathrm{HCl}$ buffer $(\mathrm{pH} 7.5-7.8), 1 \mathrm{ml}$ of a of azocasein solution was transferred into a test tube, $0.1 \mathrm{ml}$ of $0.1 \% \mathrm{CaCl} 2,0,1 \mathrm{ml}$ of an 
experimental sample (culture fluid) and incubated in a thermostat at $37{ }^{\circ} \mathrm{C}$ for $20 \mathrm{~min}$. Then, $2 \mathrm{ml}$ of $10 \%$ trichloroacetic acid (TCA) was introduced into the test tube to stop the reaction and precipitate a non-reactive substrate. The tube was kept for $30 \mathrm{~min}$ in an ice bath, the contents were centrifuged for $15 \mathrm{~min}$ at 10 thousand rpm, and $2 \mathrm{ml}$ of $0.5 \mathrm{M} \mathrm{NaOH}$ was added to $2 \mathrm{ml}$ of the supernatant. The amount of dye released as a result of protein hydrolysis was determined on a PD 303 spectrophotometer (Apel, Japan) at $440 \mathrm{~nm}$ in cuvettes with an optical path length of $1 \mathrm{~cm}$. In the control sample, instead of the experimental sample, $0.1 \mathrm{ml}$ of distilled water or buffer was added.

For a protease activity unit (E), such an amount of enzyme was taken that causes an increase in the optical density of the filtrate by 0.01 units when measured against a control sample. Protease activity was expressed in $\mathrm{U} / \mathrm{min} \mathrm{x} \mathrm{ml}$ or $\mathrm{U} / \mathrm{min} \mathrm{x} \mathrm{mg}$ protein.

\subsection{Determination of protein amount in B. subtilis P-2 cell-free culture fluid supernatant}

The protein content was determined by Bradford method. For this, $2 \mathrm{ml}$ of Bradford's reagent was added to $0.1 \mathrm{ml}$ of the analyzed sample. After $10 \mathrm{~min}$ incubation at room temperature, the optical density was measured on a spectrophotometer at a wavelength of $595 \mathrm{~nm}$. A standard albumin solution was used as a reference solution. A calibration graph was built within the concentration range from 0.01 to $1 \mathrm{mg}$ of a standard protein sample by measuring the absorbance of the solutions at $595 \mathrm{~nm}$.

\subsection{Determination of antagonistic activity of biocomposite}

Antimicrobial activity of $\mathrm{BC} / \mathrm{Ch} /$ Bacillus postbiotics was studied against Gram-negative E.coli ATCC 8799, P.aeruginosa ATCC 9027 and Gram-positive S.aureus ATCC 6538 and S.epidermidis ATCC 14990 bacteria test cultures. The antimicrobial assessment was carried out by the«agar diffusion» method. The sterile Müller-Hinton nutrient medium was poured into sterile Petri dishes with a $4 \mathrm{~mm}$ thick layer. The plates were left at room temperature to solidify. Then a suspension of the test microorganisms (inoculum) was prepared. For that purpose a pure daily culture grown on a solid nutrient medium was used. Identical, clearly isolated colonies were selected. The loopful of the cells from a single colony was transferred to a test tube with sterile saline solution and the inoculum turbidity was adjusted to a McFarland standard 0.5 , which corresponds to $1.5 \times 10^{8} \mathrm{CFU}$ (colony forming units) in $1 \mathrm{ml}$. The $2 \mathrm{ml}$ inoculum pipetted on the surface of nutrient medium in a Petri dish was evenly spread over the surface of agar by shaking, then the excess liquid was removed. The opened cups were left at a room temperature for 10 minutes for drying. $\mathrm{BC} / \mathrm{BS}$ films were placed on the surface of the nutrient medium. After the application of the biocomposite, the Petri dishes were incubated at $37{ }^{\circ} \mathrm{C}$ for 24 hours, then the growth inhibition zone of the target microorganism was measured.

The antagonistic activity of Bacillus culture fluid was determined by the «spot-on-lawn assay with wells». An agar well is prepared with metal or glass cylinders of a certain size (58) $\mathrm{mm}$. The acellular supernatantis pipetted into the wells and allowed to settle.

The agar surface should be previously inoculated with a microbial suspension giving a uniform lawn. Antagonistic activity was considered zero when the width of zone inhibition growth of test strains was up to $1 \mathrm{~mm}$, low $-1-5 \mathrm{~mm}$, medium - 5-9 mm, high - at $9 \mathrm{~mm}$ or more.

\subsection{Wound Healing Studies}

\subsubsection{Animals}


The study was carried out on male mongrel rats weighing 180-200 g. The animals were kept on a standard ration of vivarium. The animal study was carried out abiding by the national regulations related to the conduct of experimentation. The experiments were guided by the "Rules on work with experimental animals". Three groups of male rats, five in each received the following treatment schedule: I - was covered with $\mathrm{BC} / \mathrm{Ch} /$ Bacillus subtilis postbiotics biocomposite; II - was closed with a BC film; III - was served as the control (just cleaning the wounds with a physiologic serum). After the wounding process, each rat was housed in a sterilized cage and given autoclaved food and redistilled water in order to prevent bacterial infection.

\subsubsection{Induction of wounds}

The wound healing activity of BC/BS biocomposite was evaluated on excision wound rats models. After total anesthesia with ketamine $(100 \mathrm{mg} / \mathrm{kg}$ body weight $)$ by intramuscular injection, a $2.0 \mathrm{~cm}$ longitudinal full thickness incision was made in the back of each rat. An excision wound margin was traced after wound creation by using graph paper and size was calculated. Wound contraction was evaluated on 3, 5, 7, 14 and 21 days until complete wound healing and expressed in percentage of healed wound size. The wounding day was considered as day 0 . Wound closure, considered as percentage reduction of original wound size, was determined using the following formula 1:

$$
\text { Wound closure, } \%=\frac{A 0-A d}{A 0} \times 100
$$

where $A_{0}$ was the initial wound size (day 0 ) and Ad was the wound size on present day (d). The period of epithelization was estimated as the number of days required for falling of the dead tissue without any residual raw wound.

\subsection{Statistical analysis}

Statistical comparison was performed using unpaired t-test and one-way analysis of variance (ANOVA) followed by Dunnett's test for multiple comparisons. All statistical analyses were performed using SPSS 16.0 software package (SPSS Inc., USA)

\section{Results and discussion}

The results on preparation and study of physicochemical properties of a biocomposite material based on BC and chitosan were published earlier [7]. The inclusion of chitosan in $\mathrm{BC}$ was carried out by their joint aggregation with a gel film. The obtained $\mathrm{BC} /$ chitosan film samples were homogeneous and transparent. The mass ratio of $\mathrm{BC}$ and chitosan was 75:25.

The composite material $\mathrm{BC} / \mathrm{Chitosan}$ has an interconnected porous matrix structure with a large surface area. Micro- $(15-35 \mathrm{~nm})$ and macrofibrils $(50-150 \mathrm{~nm})$ in BC and $\mathrm{BC} /$ Chitosan films are combined into ribbon-like fibers, providing a high degree of crystallinity (up to $80 \%$ ) and mechanical strength (Young's modulus: $36.03 \pm 1,80 \mathrm{MPa}$; tensile strength: $22.48 \pm 0.20 \mathrm{MPa}$ ). The chemical interaction of $\mathrm{BC}$ and chitosan in the composition of films was established by IR spectrometry.

The $\mathrm{BC}$ and $\mathrm{BC} /$ Chitosan films have a flat and smooth surface. With sufficient moisture, such films will be easily removed from wound without damaging the "fresh" epithelium. 
To obtain a biocomposite material (BC/Ch/B. subtilis exometabolites) the separation of cells from culture liquid should be provided. Separation of native microbial culture (NMC) was carried out by centrifugation. Since the culture fluid supernatant after separation contained up to $10^{4}-10^{5}$ bacterial cells per $\mathrm{ml}$, they were removed by membrane filtration (MILLIPORE filter with a pore size of $0.45 \mu \mathrm{m}$ ). Most of antibiotics produced by $B$. subtilis have protein or polypeptide nature [8]. In this regard, the presence of fermentation products with biological activity in the cell-free culture fluid supernatant of (CFS) was analyzed by three parameters: protein content, proteolytic and antagonistic activity. By the end of $B$. subtilis $\mathrm{P}-2$ cultivation (i.e., by 24 hours), the CFS accumulated up to $0.541 \pm 13.4 \mathrm{mg} / \mathrm{ml}$ of protein. The level of proteases in NMC reached $9.3 \pm 0.6 \mathrm{U} / \mathrm{ml}$, and in supernatant $-7.8 \pm$ $0.3 \mathrm{U} / \mathrm{ml}$, i.e. the level of these enzymes in supernatant is only 1.2 times lower than in native microbial culture.

To determine the antagonistic activity, $500 \mu \mathrm{l}$ of NMC and CFS of B. subtilisP-2 strain were added into wells of nutrient agar inoculated with test cultures. The level of antimicrobial activity of $B$. subtilis P-2 NMC and CFS was measured by the size of target microorganism growth inhibition zone (Figure 1).

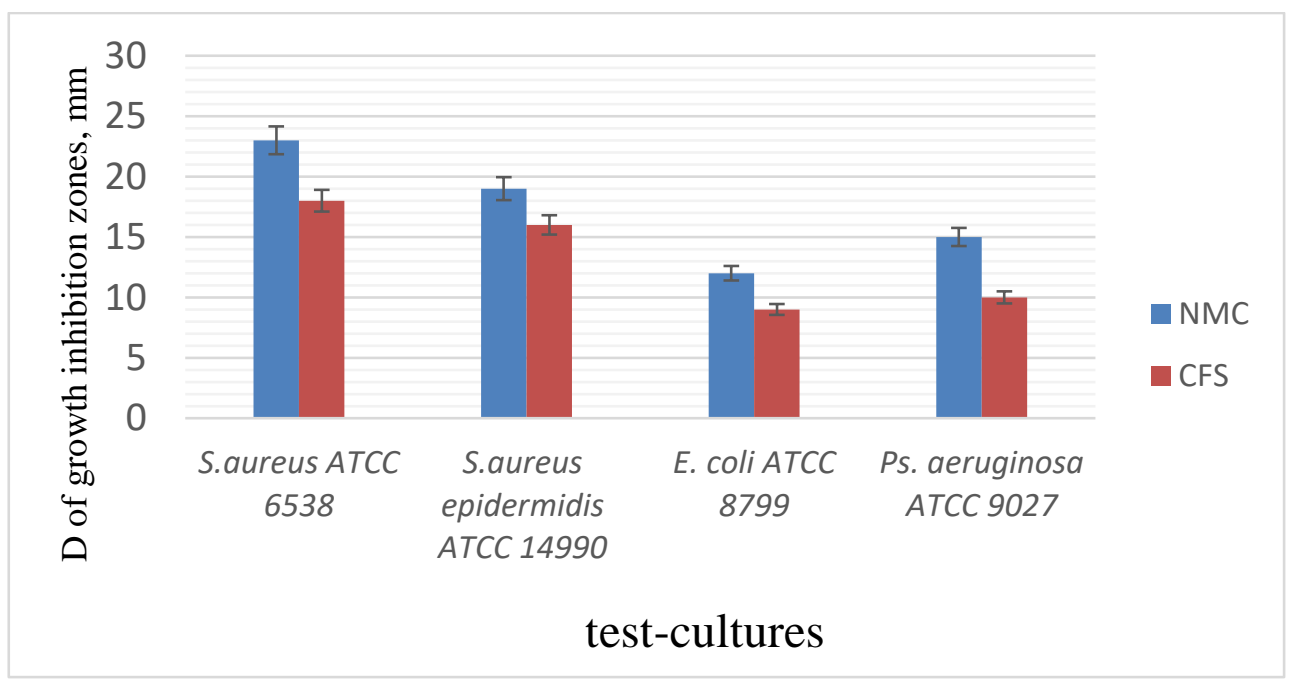

Fig. 1. Average growth inhibition zone sizes of reference bacterial strains during study of anantimicrobial action of NMC and CFS of Bacillusby «spot-on-lawn assay with wells»

NMC of Bacillus subtilis P-2 strain is characterized by a high level of antagonistic activity. However, in its culture fluid supernatant, it is almost $26 \%$ lower. Nevertheless, the level of antagonistic activity of the supernatant remains rather high, which indicates the presence of antimicrobial substances in it.

Summarizing the data obtained on determination of biological activity of CFS of $B$. subtilis $\mathrm{P}-2$, it is obvious that, in addition to antimicrobial substances, it also contains proteases. The results obtained indicate the presence of substances with target activity in it, promoting wound healing.

The immobilization of metabolites contained in B. subtilis P-2 CFS was carried out by adsorption of metabolites on bacterial cellulose film. Recently, bacterial cellulose has been often used to immobilize enzymes. For example, glucoamylases for hydrolysis of starch to glucose in bioethanol production [8]. Also, work is underway to create composites based on $\mathrm{BC} / \mathrm{Ch}$ with immobilized ureases, which are used to detoxify blood in artificial kidneys, remove urea from drinks and food products, and reduce the content of urea during wastewater treatment [9]. Adsorption immobilization converts the enzyme from among homogeneous 
soluble catalysts to heterogeneous ones, which makes it possible to increase the stability of the biocatalyst under the influence of denaturing environmental factors.

For the sorption immobilization of metabolites contained in B. subtilis P-2 CFS, pieces $\mathrm{BC}$ film were introduced into this cell-free extract and incubated at room temperature $\left(25^{\circ}\right.$ C) under dynamic conditions $(80 \mathrm{rpm})$ for 5 minutes. Then $0.5 \mathrm{ml}$ of a $2.5 \%$ glutaraldehyde solution was added and kept at $30^{\circ} \mathrm{C}$ for 2 hours at $100 \mathrm{rpm}$. To complete the immobilization, the $\mathrm{BC}$ films were washed several times with phosphate buffer $(\mathrm{pH} 5.2,200 \mathrm{mM})$ and dried [10].

The immobilization efficiency of target exometabolites (peptide antibiotics and proteolytic enzymes) on $\mathrm{BC} / \mathrm{Ch}$ was determined by the protein content and level of proteolytic enzymes in the Bacillus $\mathrm{CFS}$ and the $\mathrm{BC} / \mathrm{Ch}$ film incubated in it.

The protein content in $\mathrm{BC} / \mathrm{Ch}$ film and Bacillus CFS during incubation for 2.5 hours was determined by Bradford method. A standard albumin solution was used as a reference solution(Figure 2).

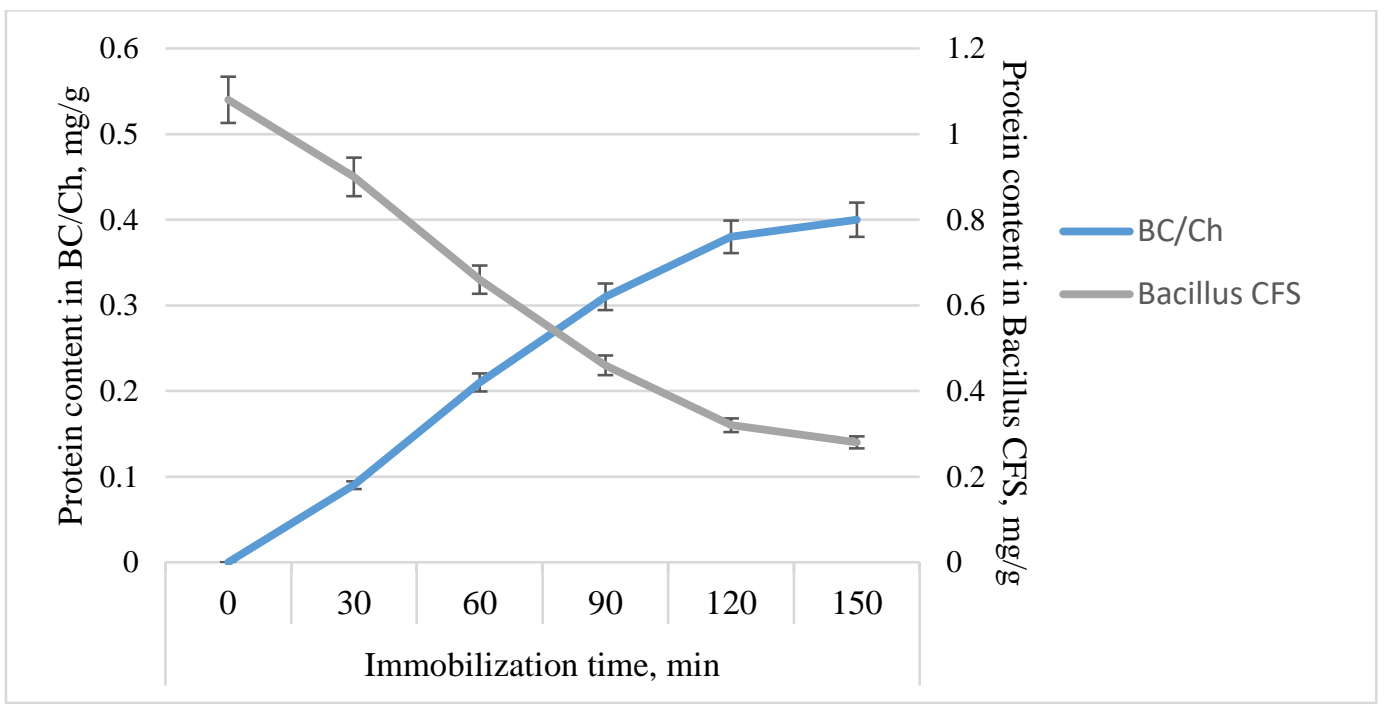

Fig.2.Sorption dynamics of protein exometabolites on the BC/Ch film

It was found that, depending on cultivation time, different protein content is concentrated in $\mathrm{BC} / \mathrm{Ch}$ nanofibers. Immobilization of protein molecules occurs after $150 \mathrm{~min}$ of incubation. The efficiency of immobilization was $74 \%$.

At the next stage, the level of protease concentration in CFS and $\mathrm{BC} / \mathrm{Ch}$ was determined during their joint incubation (Figure 3). To extract the proteases immobilized on the $\mathrm{BC} / \mathrm{Ch}$, $1 \mathrm{~g}$ of gel film was cut into pieces, and then introduced into a test tube containing a $10 \%$ solution of sodium dodecyl sulfate. Then $\mathrm{BC} / \mathrm{Ch}$ was treated with ultrasound for $20 \mathrm{~min}$ in an ice bath.

The proteolytic activity of $B$. subtilis $\mathrm{P}-2$ culture liquid was $7.8 \pm 0.6 \mathrm{U} / \mathrm{min} \mathrm{x} \mathrm{ml}$. 


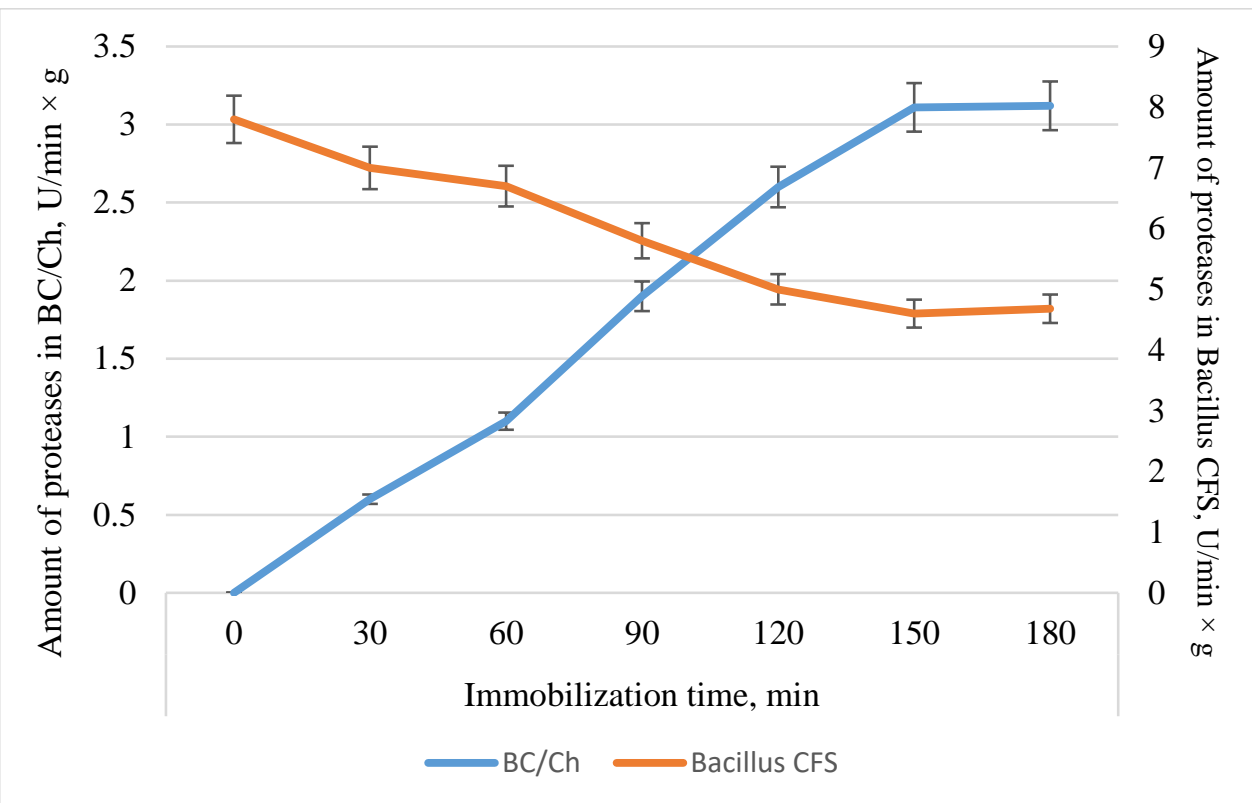

Fig. 3.Dynamics of the sorption of proteases on $\mathrm{BC} / \mathrm{Ch}$ film

The nature of changes in concentration values of proteases in cultural liquid indicates the sequential immobilization of $B$. subtilis P-2 enzymes on the BC/Ch gel film already starting from a 30-minute contact, which leads to a decrease in the amount of these enzymes in CFS and an increase in $\mathrm{BC} / \mathrm{Ch}$ respectively. After 2.5 hours of incubation, the $\mathrm{BC} / \mathrm{Ch}$ film is saturated with $B$. subtilis P-2 enzymes. Based on data obtained, it can be concluded that 150 $\mathrm{min}$ is the optimal time for immobilization of B. subtilis $\mathrm{P}-2$ proteases on a $\mathrm{BC} / \mathrm{Ch}$ gel film. In this case, the efficiency of immobilization of proteases on the $\mathrm{BC} / \mathrm{C}$ film is $40 \%$.

The antagonistic activity of $\mathrm{BC}$ composites with chitosan containing metabolites of the antagonist bacterium was determined by agar diffusion method. The sensitivity of microorganisms to biocomposite was studied using reference strains $S$. aureus, $P$. aeruginosa, E. coli, S. epidermidis. Composite material BC/chitosan/bacterial metabolites has the highest activity in comparison with non-functionalized BC (Figure 4). It worked best on Staphylococcus strains.

The film BC/Chitosan /B. subtilis exometabolites has the highest antimicrobial activity against all test strains in comparison with the non-functionalized BC. The growth inhibition zone of St. aureus strains is $31 \mathrm{~mm}$, E. coli up to $19 \mathrm{~mm}$, Ps.aeruginosa $-27 \mathrm{~mm}$, and St. epidermidis up to $28 \mathrm{~mm}$. This means that the inclusion of chitosan and B. subtilis postbiotic into bacterial cellulose leads to an increase in antibacterial efficiency of this biocomposite.

There are two main mechanisms of the antimicrobial action of chitosan, both related to the number of active amino groups. First, positively charged chitosan interacts with negatively charged bacteria, leading to an increase in permeability of bacterial membrane, which leads to inhibition of cell growth in test strains. Another mechanism involves binding of chitosan to DNA, which can suppress the production of bacterial mRNA [11].

It should be noted that antibacterial activity of $B$. subtilis postbiotic is enhanced by the action of lytic enzymes that actively lyse cells of both gram-positive and gram-negative microorganisms. Interestingly, when analyzing the microbial contamination of wounds of various etiology, it was found that the presence of B. subtilis exometabolites in them prevents the development of purulent inflammation [11]. The above data are part of the rationale for the correct choice of these components for immobilization on $\mathrm{BC}$ for wound dressing application. 


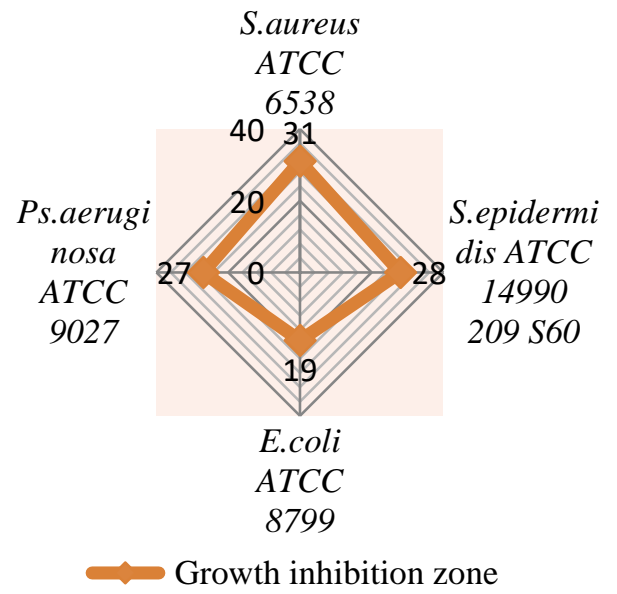

$\mathrm{BC} / \mathrm{Ch} /$ B.subtilismetabolites

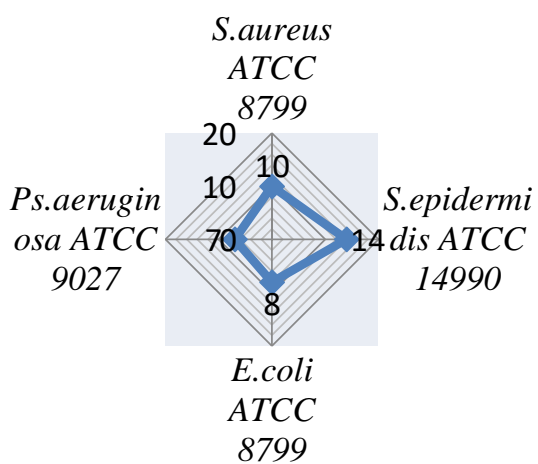

$\sim$ Growth inhibition zone

Fig. 4. Evaluation of the antimicrobial action effectiveness of materials on reference strains of microorganisms by growth inhibition zones

The bioactive wound coatings based on BC/Ch with immobilized therein cells of BS destined for the local application. The therapeutic efficacy of the investigated gel-film samples has been evaluated on reducing the wound surface size and the healing time. The results of the action of composite gel films on the healing of excision wounds are presented in Table 1.

Table 1. Effect of $\mathrm{BC} / \mathrm{Ch} /$ B.subtilis metabolites and $\mathrm{BC}$ films on wound contraction and epithelization period in excision wound

\begin{tabular}{|c|c|c|c|c|c|c|}
\hline \multirow{2}{*}{ Group of animals } & \multicolumn{5}{|c|}{ Wound size, mm } \\
\cline { 2 - 7 } & $\mathbf{0}^{\text {th }}$ day & $\mathbf{3}^{\text {rd }}$ day & $\mathbf{5}^{\text {th }}$ day & $\mathbf{7}^{\text {th }} \mathbf{d a y}$ & $\mathbf{1 4}^{\text {th }} \mathbf{d a y}$ & $\mathbf{2 1}^{\text {st }}$ day \\
\hline $\begin{array}{c}\text { I - BC/Ch/B.subtilis } \\
\text { metabolites }\end{array}$ & $191 \pm 3^{\mathrm{a}}$ & $94 \pm 2^{\mathrm{b}}$ & $27 \pm 3^{\mathrm{b}}$ & 0 & 0 & 0 \\
\hline II - BCfilm & $188 \pm 4^{\mathrm{a}}$ & $105 \pm 7^{\mathrm{b}}$ & $53 \pm 2^{\mathrm{c}}$ & $14 \pm 2^{\mathrm{c}}$ & 0 & 0 \\
\hline $\begin{array}{c}\text { III - Control(without } \\
\text { treatment) }\end{array}$ & $192 \pm 5^{\mathrm{a}}$ & $175 \pm 4^{\mathrm{a}}$ & $105 \pm 7^{\mathrm{a}}$ & $64 \pm 3^{\mathrm{a}}$ & $47 \pm 2^{\mathrm{a}}$ & $3.2 \pm 0.9^{\mathrm{a}}$ \\
\hline $\begin{array}{l}\text { Note: Values are mean } \pm \text { SEM (Percent) of 5 rats in each group. A } P<0.05, \mathrm{~b} P<0.01, \text { and c } P< \\
\text { 0.001 compared to respective day control group (statistical analysis was done by one-way analysis } \\
\text { of variance followed by Dunnett's test for multiple comparisons). }\end{array}$ \\
\hline
\end{tabular}

Based on the data obtained, the greatest wound healing activity has been reached with the biocomposite containing chitosan and BS metabolites included in BC film. In this group of rats, the time of complete healing have been 7 days in all animals. Somewhat slower, on day 14 , the wound have been healed with the application of the "non-functionalized" cellulose gel film. It is submitted that the wounds are quickly covered with epithelium in a moist environment [1]. BC have a good absorbing capacity and optimal permeability for exudate, 
which provides the conditions for facilitating wound healing [12]. The use of all experimental variants led to an accelerated recovery of the wound surface in comparison with the control group without treatment (21 days).

During the first two days, there was a large amount of fluid accumulated in wounds. However, all samples of films absorbed it efficiently. It should be noted that the replacement of wound dressings did not cause damage to the regenerated wounds, since the films were easily detached from the wound surface. On the seventh to twenty first day, a new epidermis formed on the affected areas, i.e. epithelialization occurred, and the wounds healed. No inflammation was observed during healing.

In addition to registred changes in the wound area, the course of healing was analyzed by a number of indicators. Thus, with visual observation, the time of granulations appearance in the wound, closure of wound with granulations, filling of wound with granulations, the quality of granulations were recorded, and the usefulness of epithelialization and condition of tissues around the wound were also assessed.

So, on days 4-5 in the group of animals treated with an experimental composite sample based on $\mathrm{BC} / \mathrm{Ch} /$ Bacillus metabolites, the local inflammatory reaction decreased, on days 47 the formation of granulation tissue began, on days 6-8 the wound was completely cleared of necrotic tissues and epithelialization began. Complete healing occurred within 7 to 9 days, versus 21 days in the control (without treatment) group and 14 days in the group of animals, where non-functionalized cellulose gel film for wound treatment was used.

A decrease in duration of wound removal in experimental groups of animals treated with experimental samples of films based on $\mathrm{BC} / \mathrm{Ch} / \mathrm{B}$. subtilis metabolites occurred $20 \%$ faster when compared with the control (without treatment) group of animals.

The therapeutic effect is due to the complex action of the functional ingredients included in the biocomposite material: postbiotic in combination with chitosan. The developed bioactive wound dressings are intended for local application in order to optimize the wound healing process.

\section{Conclusion}

The biocomposite based on BC gel film, chitosan and BS postbiotic was obtained. Antibacterial activity of the composite is enhanced by the lytic enzymes action actively lysing cells of both gram-positive and gram-negative bacteria - the typical causative agents of wound infections.

The obtained biocomposite has a high therapeutic efficiency on wound process models. All these factors together can shorten the time needed for treatment of wounds. Overall, these results suggest the promising application of the biocompositeas a wound dressing, to promote full-thickness wound healing.

\section{References}

1. N. Shah, M. Ul-Islam, WA. Khattak, JK. Park Carbohydr. Polym. 98, 2 (2013).

2. Czaja, W. K.., Young, D. J., Kawecki M., Brown R. M., Biomacromolecules. 8, 1(2007).

3. L. R. Lynd, P. J. Weimer, W. H van Zyl., I. S Pretorius., Microbiology and Molecular Biology Reviews. 66, 3 (2002).

4. C. Lin, C.Lien, H.Yeh, C.Yu, S.Hsu Carbohydrate polymers. 94 (2013).

5. M. Rosewald, Y. Fang, M.Tamutsiva, A.Harkins, C Tran ECS Transactions. 64, 4 (2014). 
6. V.V.Smirnov, S.R. Reznik, V.A. Vyunitskaya, Journal of Microbiology. 24, 4 (2002).

7. I.S. Savitskaya, A.S.Kistaubayeva, I.E. Digel, D.H. Shokatayeva, Eurasian ChemicoTechnological Journal, 19(3) (2017).

8. W. Sheng-Chi, L.Ying-Ke, H. Chia-Yen International journal of science and engineering, 3, 4 (2013).

9. M. Pesaran, Gh. Amoabediny, F. Yazdian, International journal of polymer science. 14, 7 (2015).

10. W. Sheng-Chi, L.Ying-Ke, Journal of molecular catalysis B: Enzymatic, 54, 4 (2008).

11. E. Rohaeti, W. Endang, A. Rakhmawati, JurnalIlmia Kimia, 11, 1 (2016).

12. N.A. Zabokritskiy, The Journal of scientific articles "Health and education millennium"16, 4 (2014). 